\title{
Upregulation of the miR-17-92 cluster and its two paraloga in osteosarcoma - reasons and consequences
}

\author{
Leila Arabi ${ }^{1,2, *}$, Joël R Gsponer ${ }^{1, *}{ }^{,}$Jan Smida $^{3}$, Michaela Nathrath $^{3}$, Valeria Perrina ${ }^{1}$, \\ Gernot Jundt $^{1,4}$, Christian Ruiz ${ }^{1}$, Luca Quagliata ${ }^{1,5}$, and Daniel Baumhoer ${ }^{1,4,5}$ \\ ${ }^{1}$ Institute of Pathology, University Hospital Basel, Basel, Switzerland; \\ ${ }^{2}$ Nanotechnology Research Center, School of Pharmacy, Mashhad University of Medical Sciences, Mashhad, Iran; \\ ${ }^{3}$ Clinical Cooperation Group Osteosarcoma, Helmholtz Zentrum Muenchen, German Research Center for Environmental \\ Health, Neuherberg, Germany; \\ ${ }^{4}$ Bone Tumor Reference Center at the Institute of Pathology, University Hospital Basel, Basel, Switzerland \\ ${ }^{5}$ Shared senior authorship \\ * The authors contributed equally to this work
}

Correspondence to: Daniel Baumhoer, email: dbaumhoer@mac.com

Keywords: osteosarcoma, miR-17-92, miR-106a-363, miR-106b-25, FAS, BIM

Received: March 11, $2014 \quad$ Accepted: May 06, $2014 \quad$ Published: May 06, 2014

This is an open-access article distributed under the terms of the Creative Commons Attribution License, which permits unrestricted use, distribution, and reproduction in any medium, provided the original author and source are credited.

\section{ABSTRACT:}

Osteosarcomas (OS) are aggressive bone tumors characterized by complex karyotypes with highly variable structural and numerical chromosomal aberrations. Although several genes and pathways commonly altered in malignant tumors have also been identified in $0 S$, the molecular pathogenesis and driving genetic events eventually leading to tumor development are still poorly understood. The microRNA (miRNA) cluster 17-92 and its two paraloga 106a-363 and 106b-25 are known to have diverse oncogenic properties and have been shown to be constantly upregulated in several established OS cell lines. In this study we analyzed a series of $\mathbf{7 5}$ well characterized pretherapeutic OS samples for their expression of cluster-related miRNAs and correlated our findings with clinico-pathological parameters including prognosis, metastases and response to neoadjuvant therapy. Interestingly, higher expression levels of specific miRNAs were significantly associated with an adverse outcome of patients and were also higher in patients with systemic spread. We could furthermore show a direct correlation between the expression of cluster activators (MYC, E2F1-3), inhibitors (TP53), individual miRNAs, and pro-apoptotic targets (FAS, BIM). Our findings therefore underline a critical role of the miR-17-92 cluster and its two paraloga in OS biology with pathogenetic and prognostic impact.

\section{INTRODUCTION}

Osteosarcomas (OS) are the most common primary malignant tumors of bone generally affecting the metaphyses of long bones in children and adolescents [1]. Due to a high rate of systemic spread already at the time of diagnosis patients greatly benefit from (neo-) adjuvant polychemotherapy in addition to radical surgery and reach 10 -year survival rates of up to $73 \%$ in case of good response to cytostatic regimens [2, 3]. However, a substantial group of patients with metastatic, recurrent and/or refractory disease still lacks effective treatment options underlining the urgent need for new therapeutic alternatives and targets. Furthermore, there are no established biomarkers in OS that could identify patients with particularly aggressive tumors and could therefore constitute a basis for a more individualized treatment stratification [4]. One reason for this phenomenon is the genetic heterogeneity and complexity that is characteristic for OS and which hampers the identification of initiating and/or sustaining oncogenetic drivers. Amongst the most commonly mutated and/or altered genes in OS, TP53 and MYC have been identified, both of which are known to be deregulated in a variety of malignant tumors $[1,4]$. 
Besides conventional oncogenes and tumor suppressors, microRNAs (miRNA) have increasingly been recognized as regulators of gene expression that can acquire oncogenic potential. The miR-17-92 cluster, also named oncomir-I, and its two paraloga miR-106a-363 and miR-106b-25 were among the first families of those small RNA molecules that were found to be upregulated in several malignant tumors. Meanwhile, several cluster-related miRNAs were shown to accelerate tumor development, to induce angiogenesis, to prevent apoptosis, and, only recently, to crucially influence osteoblastic proliferation and differentiation [5-7]. All three clusters are part of elaborate regulatory networks and can influence the expression of various genes involved in cell cycle control, apoptosis and angiogenesis (Figure 1). Interestingly, MYC is known to stimulate the expression of cluster-related miRNAs whereas TP53 seems to have an inhibitory effect $[8,9]$. In a previous study we demonstrated the upregulation of several of the respective miRNAs in a panel of established OS cell lines (HOS58, U2-OS, Saos-2, MNNG/HOS, SJSA-1, and MG-63) which was meanwhile confirmed by an independent group $[10,11]$.

In this study, we assembled a series of well characterized pretherapeutic OS samples to validate our cell line results in tumor biopsies and to analyze if the expression of individual miRNAs correlated with clinicopathological parameters including prognosis, metastatic disease and/or response to therapy. In a next step, we interrogated the expression of selected regulators (MYC, TP53, E2F1, E2F2, E2F3) and pro-apoptotic targets (FAS, $\mathrm{BIM}$ ) of the miR-17-92 cluster and its two paraloga that have already been described to show an altered expression and potential pathogenetic impact in OS $[1,4,12,13]$. By this means, we aimed to confirm the upregulation of cluster related miRNAs and to provide a deeper insight into the causes and consequences of cluster activation in human osteosarcoma.

\section{RESULTS}

\section{Patients' and samples' characteristics}

All patients' characteristics are presented in Table 1. MicroRNA stability is known to efficiently enable expression analyses in a variety of tissue sources. In this study we extracted miRNAs from 75 formalin fixed paraffin embedded (FFPE) pretherapeutic osteosarcoma samples. The expression levels of all miRNAs were evaluable in 57/75 cases and of selected regulators and targets of the respective miRNA clusters in 41/75 cases (Table 1). Causes of samples exclusion were either insufficient amounts of tissue or poor RNA quality.

\section{MicroRNA expression in osteosarcoma}

To date no comprehensive evaluation of the miR-1792 cluster and its two paraloga has been carried out in OS. Our work revealed that several cluster-related miRNAs were constantly upregulated in the tumors analyzed compared to both human osteoblasts and mesenchymal stem cells (Figure 3a). Importantly, higher expression levels of various miRNAs were significantly associated with an adverse overall survival: miR-19a $(p=0.037)$, miR-20a ( $=0.024)$, miR-19b ( $=0.031)$, miR-92a (p $=0.006)$, and miR-106b $(p=0.032)($ Figure $3 b$ and $3 c)$. A similar trend could be shown also for all the residual miRNAs but it did not reach statistical significance (data not shown). We further investigated a potential correlation of the miRNAs with metastatic spread and with response to neoadjuvant chemotherapy. For all miRNAs analyzed, metastatic OS showed higher expression levels of the respective miRNAs than tumors without systemic spread (Figure 3d). However, statistical significance was
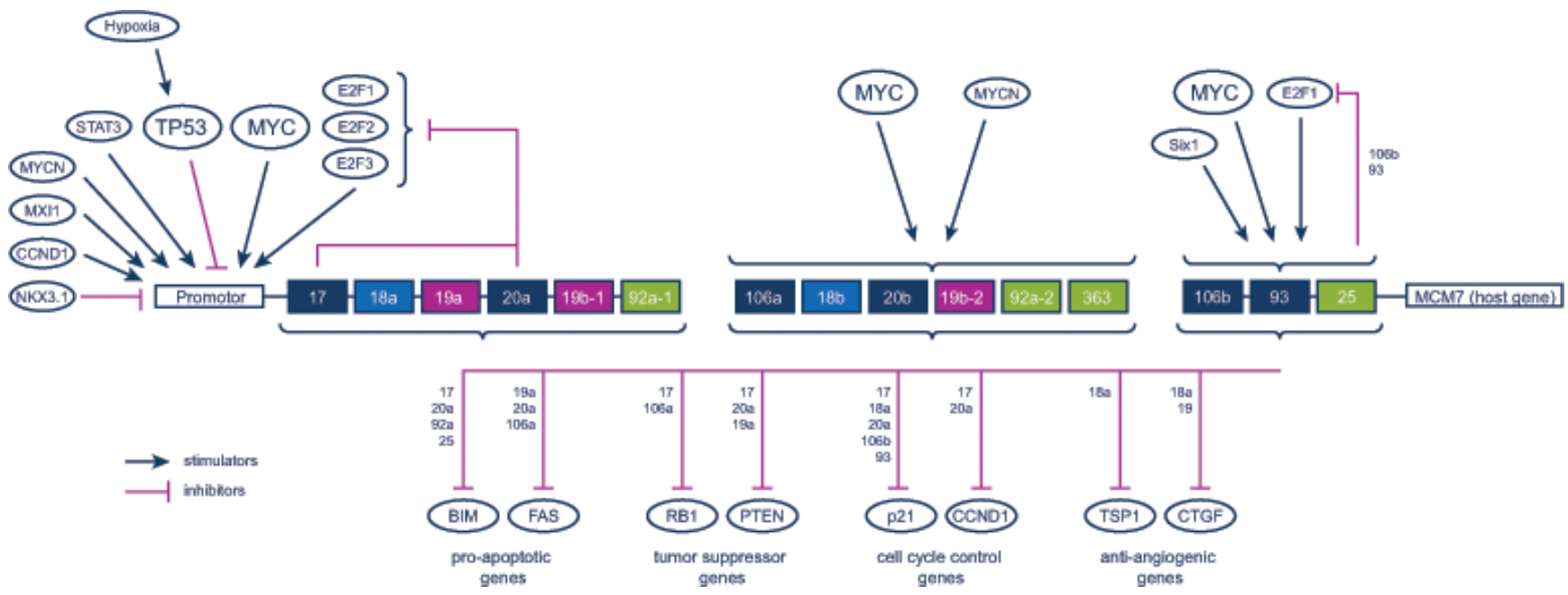

Figure 1: The miR-17-92 cluster and its two paraloga miR-106a-363 and miR-106b-25 are centered in a complex network of regulators (upper half) and targets (lower half) of which this scheme only shows a selection for a better overview $[8,9]$. 
Table 1: Patients' characteristics

\begin{tabular}{|c|c|c|c|}
\hline & Complete series $(n=75)$ & miRNA series $(n=57)$ & $\begin{array}{l}\text { Regulator / target } \\
\text { series }(n=41)\end{array}$ \\
\hline Gender & $75 / 75(100 \%)$ & $57 / 57(100 \%)$ & $41 / 41(100 \%)$ \\
\hline male & 38 & 29 & 21 \\
\hline female & 37 & 28 & 20 \\
\hline Age at diagnosis (years) & $75 / 75(100 \%)$ & $57 / 57(100 \%)$ & $41 / 41(100 \%)$ \\
\hline average & 23.5 & 24.9 & 23.8 \\
\hline median & 16 & 16 & 16 \\
\hline range & $7-82$ & $7-82$ & $7-79$ \\
\hline Metastastatic spread & $75 / 75(100 \%)$ & $57 / 57(100 \%)$ & $41 / 41(100 \%)$ \\
\hline yes & 22 & 15 & 11 \\
\hline no & 53 & 42 & 30 \\
\hline Observation period (months) & $75 / 75(100 \%)$ & $57 / 57(100 \%)$ & $41 / 41(100 \%)$ \\
\hline average & 34.6 & 27.4 & 35.2 \\
\hline median & 18 & 15 & 20 \\
\hline range & $0-142$ & $0-112$ & $0-117$ \\
\hline Response to treatment & $49 / 75(65 \%)$ & $37 / 57(64.9 \%)$ & $26 / 41(63.4 \%)$ \\
\hline good & 25 & 19 & 12 \\
\hline poor & 24 & 18 & 14 \\
\hline Recurrence & $75 / 75(100 \%)$ & $57 / 57(100 \%)$ & $41 / 41(100 \%)$ \\
\hline yes & 15 & 11 & 7 \\
\hline no & 60 & 46 & 34 \\
\hline Survival & $75 / 75(100 \%)$ & $57 / 57(100 \%)$ & $41 / 41(100 \%)$ \\
\hline alive & 62 & 46 & 34 \\
\hline deceased & 13 & 11 & 7 \\
\hline Location & $75 / 75(100 \%)$ & $57 / 57(100 \%)$ & $41 / 41(100 \%)$ \\
\hline Femur & 32 & 23 & 18 \\
\hline Tibia & 17 & 12 & 8 \\
\hline Jaws & 7 & 7 & 5 \\
\hline Humerus & 5 & 5 & 3 \\
\hline Fibula & 5 & 2 & 2 \\
\hline Pelvis & 4 & 4 & 3 \\
\hline Spine & 4 & 4 & 2 \\
\hline Ulna & 1 & - & - \\
\hline
\end{tabular}




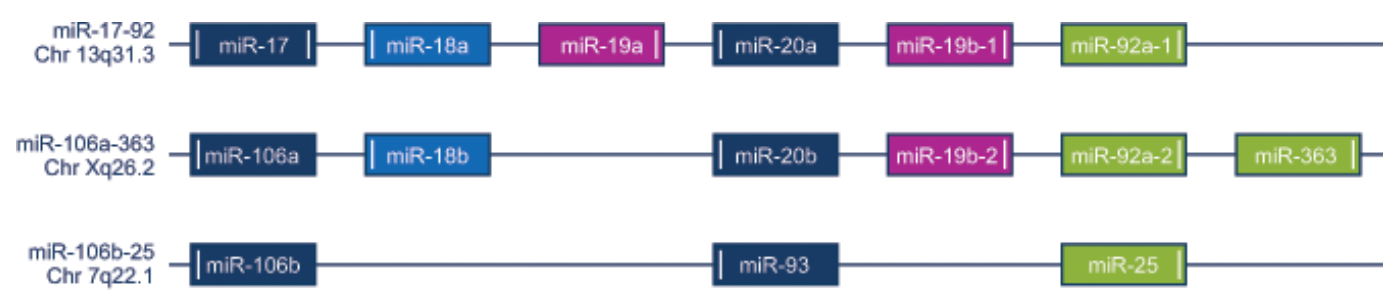

\begin{tabular}{|c|c|c|}
\hline miR-17 family & $\begin{array}{l}\text { hsa-miR-17-5p } \\
\text { hsa-miR-20a-5p } \\
\text { hsa-miR-20b-5p } \\
\text { hsa-miR-106a-5p } \\
\text { hsa-miR-106b-5p } \\
\text { hsa-miR-93-5p }\end{array}$ & $\begin{array}{l}\text { C-AAAGUG-CUUACAGUGCAGGUAG } \\
\text { U-AAAGUG-CUUAUAGUGCAGGUAG } \\
\text { C-AAAGUG-CUCAUAGUGCAGGUAG } \\
\text { A-AAAGUG-CUUACAGUGCAGGUAG } \\
\text { U-AAAGUG-CUGACAGUGCAGAU } \\
\text { C-AAAGUG-CUGUUCGUGCAGGUAG }\end{array}$ \\
\hline miR-18 family & $\begin{array}{l}\text { hsa-miR-18a-5p } \\
\text { hsa-miR-18b-5p* }\end{array}$ & $\begin{array}{l}\text { U-AAGGUG-CAUCUAGUGCAGAUAG } \\
\text { U-AAGGUG-CAUCUAGUGCAGUUAG }\end{array}$ \\
\hline miR-19 family & $\begin{array}{l}\text { hsa-miR-19a-3p } \\
\text { hsa-miR-19b-1-3p } \\
\text { hsa-miR-19b-2-3p }\end{array}$ & $\begin{array}{l}\text { U-GUGCAA-AUCUAUGCAAAACUGA } \\
\text { U-GUGCAA-AUCCAUGCAAAACUGA } \\
\text { U-GUGCAA-AUCCAUGCAAAACUGA }\end{array}$ \\
\hline miR-92 family & $\begin{array}{l}\text { hsa-miR-92a-1-3p } \\
\text { hsa-miR-92a-2-3p } \\
\text { hsa-miR-25-3p } \\
\text { hsa-miR-363-3p* }\end{array}$ & $\begin{array}{l}\text { U-AUUGCA-CUUGUCCCGGCCUGU } \\
\text { U-AUUGCA-CUUGUCCCGGCCUGU } \\
\text { C-AUUGCA-CUUGUCUCGGUCUGA } \\
\text { A-AUUGCA-CGGUAUCCAUCUGUA }\end{array}$ \\
\hline
\end{tabular}

Figure 2: The miRNA clusters are transcribed in polycistronic transcripts and organized in four families with high sequence homology $(*=$ not included in the analysis).
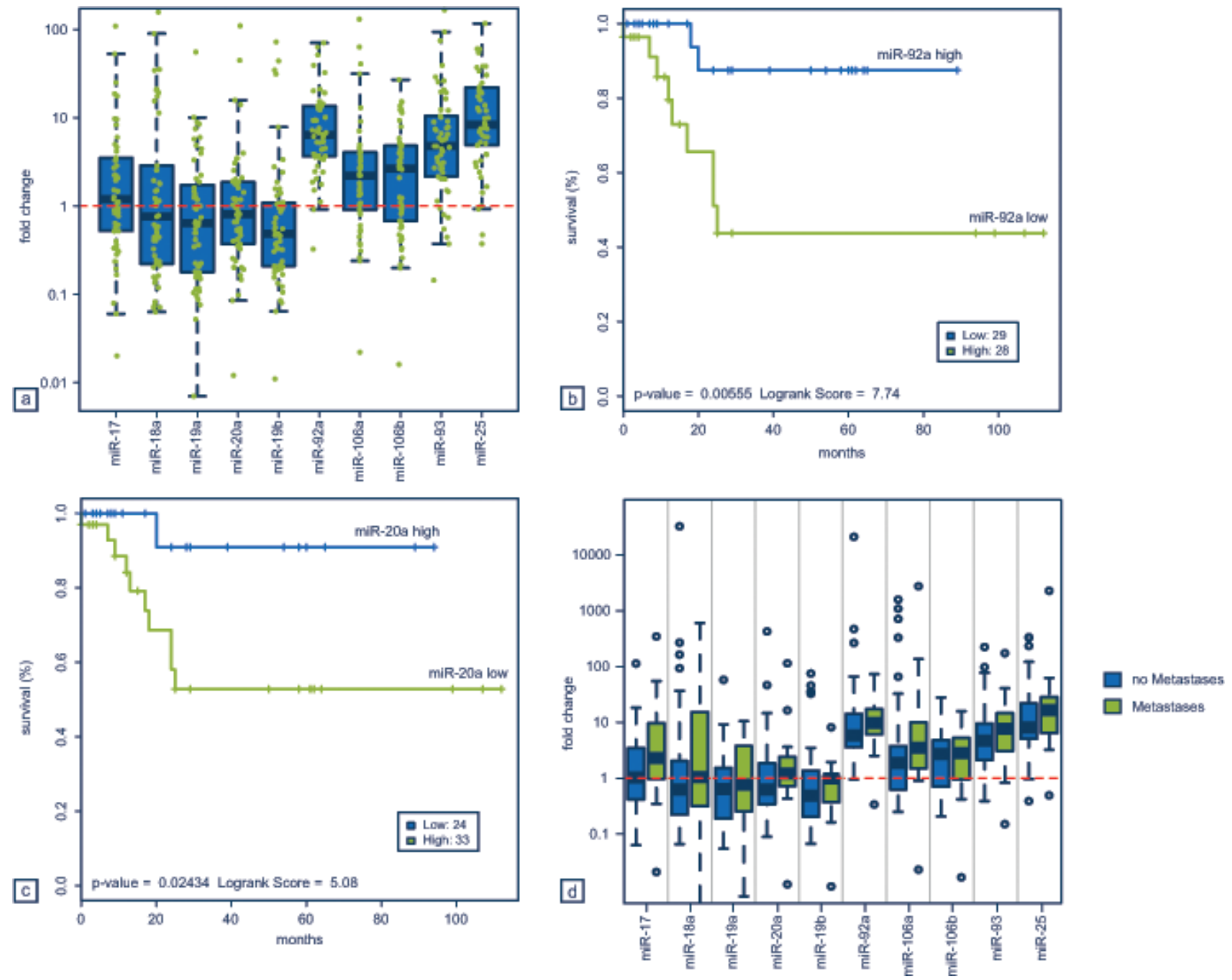

Figure 3: Relative miRNA expression of all analyzed tumors compared to the corresponding expression in L87/4 cells (a). Kaplan-Meier plots showing an adverse outcome for OS patients with higher expression levels miRNAs miR-92a (b) and miR-20a (c). Statistical significance was assessed by means of the logrank test. Relative miRNA expression of all analyzed tumor stratified by metastatic spread (d). 
not reached. There was no correlation between higher expression levels of cluster-related miRNAs and a poor response to chemotherapy (data not shown).

\section{Expression of selected regulators}

MicroRNAs are generally centered in complex networks of regulatory genes complicating the possibility to identify direct partner interactions. Taking advantage of previous work, we have selected a number of potential direct regulators of the miR-17-92 cluster and its two paraloga and analyzed their expression in our cohort of osteosarcoma samples. As expected, higher expression levels of MYC, E2F1, E2F2, and E2F3 showed a trend towards concomitant higher expression levels of most of the analyzed miRNAs, in line with the concept of those genes to represent stimulators of the respective clusters (Figure 4a). For TP53 as an inhibitor of the clusterrelated miRNAs, the opposite effect was observed in the vast majority of cases (Figure 4b). However, statistical significance was only detected for E2F1 / E2F2 and miR25 as well as for E2F3 and miR-20a / miR-93 (data not shown).

\section{Expression of selected Targets}

Driven by sequence specific targeting, miRNAs have been shown to influence a plethora of different genes. Here we exemplarily tested whether the expression levels of cluster-related miRNAs could be correlated with predicted and pro-apoptotic target genes that are known to be frequently downregulated in osteosarcoma. Indeed,
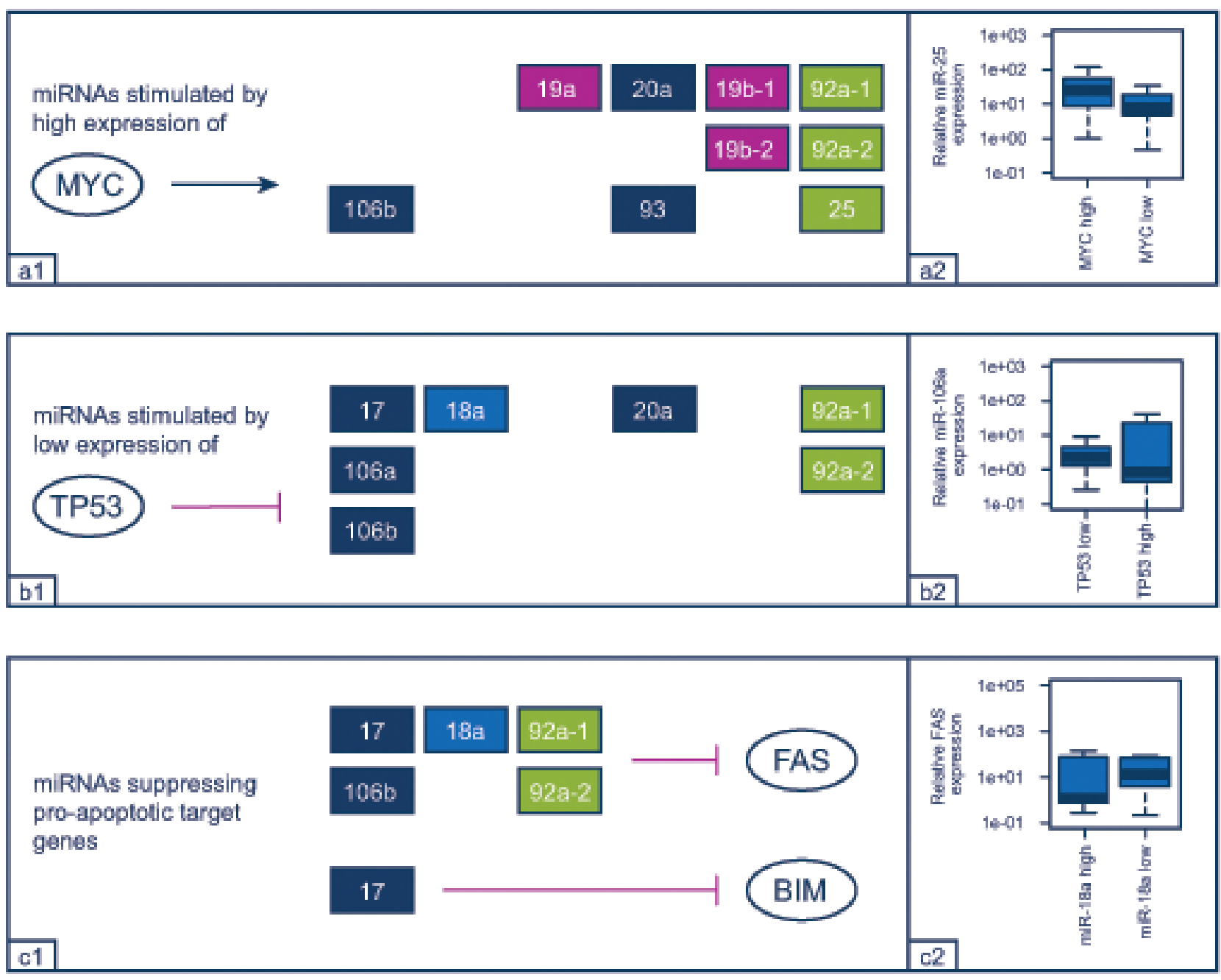

Figure 4: Comparison between regulator / target gene and miRNA expression levels. Higher expression levels of MYC showed a trend towards concomitant higher expression levels of the depicted miRNAs (a1) which is presented exemplarily for miR-25 in a separate box-plot (a2). Representing a cluster inhibitor, lower expression levels of TP53 correlated with higher expression levels of the presented miRNAs (b1). Again, miR-106a is shown exemplarily in a separate box plot (b2). Higher expression levels of the miRNA depicted revealed a trend towards concomitant lower expression levels of the pro-apoptotic targets FAS and BIM (c1). Exemplarily, the association between FAS and miR-18a expression is shown in a separate box plot (c2). 
higher expression levels of some miRNAs (miR-17, miR18a, miR-92a, and miR-106b) showed a trend towards downregulation of FAS. For BIM, only miR-17 revealed a similar effect (Figure 4c). None of those inversed relations reached statistical significance.

\section{DISCUSSION}

In the series of pretherapeutic OS samples analyzed in the present study, several members the miR-17-92 cluster and its two paraloga were constantly upregulated in the vast majority of tumors and the expression levels of individual miRNAs correlated with an adverse outcome of patients and metastatic spread. Amplification or elevated expression of the cluster-related miRNAs has previously been shown in various other malignant tumors and has also been linked to an unfavourable prognosis of patients $[8,20]$. However, this is the first study revealing a prognostic impact of these miRNA clusters in human OS. Since metastatic spread is the most adverse prognostic factor in OS, elevated miRNA expression associated with both prognosis and metastases is not surprising [1]. The response to neoadjuvant chemotherapy was nonetheless not correlated to miRNA expression levels which might be influenced by the relatively low number of cases for which this information was available (Table 1).

The regulators stimulating and suppressing the cluster-related miRNA as well as the targets of the individual miRNAs form an elaborate network of genes and pathways commonly deregulated in cancer (Fig. 1) [8, 9]. Since many functional interdependencies and feedback loops are involved, the identification of direct relations and interactions between isolated network members is difficult to accomplish. For this study, we chose known stimulators (MYC, E2F1-3) and inhibitors (TP53) of the miRNA clusters that are frequently amplified, elevated or inactivated in OS and could show that all of those regulators correlated with an up- or downregulation of the corresponding miRNAs in most of the cases as anticipated. Moreover, MYC amplification has been shown to represent an adverse prognostic factor in OS as our results also point out for several miRNAs stimulated by MYC [21]. TP53 is affected by germ line mutations, LOH or deletions in approximately $40 \%$ of OS and although the expression level of TP53 can certainly not reflect the complete functional status of the gene, tumors with low expression of TP53 showed relatively higher levels of the respective miRNAs. Importantly, the comparison of expression levels can only indicate but not definitely proof an interplay between regulators and miRNAs. On the other hand, some of the most commonly altered genes in OS all seem to participate in stimulating the expression of the miR-17-92 cluster and its two paraloga providing a rationale for further investigation.

In a recent work by Huang and colleagues, higher expression levels of miR-20a were shown to suppress
FAS in several OS cell lines and were associated with higher rates of metastatic spread in a mouse model [13]. Similarly, in gastric cancer, inhibition of FAS-mediated apoptosis was linked to an overexpression of the clusterrelated miR-106a which was shown in cell lines but also in FFPE samples [22]. In our series, we found that higher expression levels of miR-17, miR-18a, miR-92a, and miR-106b correlated with lower levels of FAS. Since the respective miRNA clusters are transcribed in polycistronic transcripts and organized in four different families with high sequence homology, our results might indicate a certain amount of interchangeability of the cluster-related miRNAs as miR-20a and miR-106a are part of the same miRNA-family like miR-17 and miR-106b (Fig. 2). BIM represents another gene with pro-apoptotic properties that is suppressed by the miR-17-92 cluster in various tumors but also in osteoblasts [23]. In our series, only miR-17 was correlated with a downregulated BIM expression.

Understanding the molecular pathogenesis of OS including the reasons for tumor development, mechanisms of systemic spread and primary chemoresistance in a subgroup of patients is the prerequisite for a more individualized treatment stratification and for identifying urgently needed new and innovative therapeutic targets. The highly variable genetic alterations found in OS which at least in a subgroup of tumors results from chromothripsis with random chromosomal rearrangements, makes driving genetic events difficult to determine [24, 25]. Several genes and pathways involved and altered in OS, however, seem to be part of the regulatory network the miR-17-92 cluster and its two paraloga miR-106a-363 and miR-106b-25 are centred in $[1,4,8,9]$. RB1 for example is commonly mutated in OS but miRNAmediated silencing of the gene might be an alternative mechanism to inactivate the tumor suppressor properties of RB1 [26, 27]. The constant upregulation of the clusterrelated miRNAs as shown here might therefore indicate a superordinate role of the clusters in OS biology and a point of intersection at which several oncogenetic pathways and events converge. Further functional studies are needed to prove if an upregulation of cluster-related miRNAs is indeed mandatory for OS development and progression and therefore might represent a new therapeutic target as was proposed recently [28]. The compelling prognostic impact of elevated miRNA expression shown here, however, clearly points to a central pathogenetic role of the three miRNA clusters in OS biology.

\section{MATERIAL \& METHODS}

\section{Tumor samples and cell lines}

75 cases of pretherapeutic OS samples with detailed clinico-pathological data were selected from the archives 
of the Bone Tumor Reference Center in Basel. Clinicopathological data included follow-up, rates of metastatic spread and recurrent disease as well as response to neoadjuvant treatment.

Since there is no normal or adjacent tissue that OS are derived from and most likely mesenchymal stem cells with at least some extent of osteoblastic differentiation represent the source of tumor development, cultured human osteoblasts (hFOB 19.9) and mesenchymal stem cells (L87/4) were used as normal controls. Cells were grown and subsequently transferred into paraffin blocks according to routine protocols to ensure similar conditions for further workup as for the tumor samples.

\section{MicroRNA extraction}

MicroRNAs extraction was performed using the High Pure miRNA Isolation Kit (Roche, Switzerland) according to the manufacturer's instructions. RNA purity and quantity were measured using the 2100 Bioanalyzer (Agilent Technologies, Germany).

\section{Quantitative Real-time Reverse-transcription polymerase chain reaction}

The expression levels of various miRNAs included in the miR-17-92 cluster and its two paraloga miR106a-363 and miR-106b-25 (Figure 2) were determined using miRCURYTM LNA Universal RT microRNA PCR analysis (Exiqon, Denmark). The two-step protocol involved universal reverse transcription (RT) to convert miRNAs into complementary DNA, followed by realtime quantitative PCR amplification using microRNAspecific and $\mathrm{LNA}^{\mathrm{TM}}$-enhanced forward and reverse primers. The small non-coding RNA SNORD44 was used as an endogenous control and NEW ExiLENT SYBR ${ }^{\circledR}$ Green was applied for PCR product detection and quantification (Exiqon, Denmark). All samples were analyzed in triplicates. To calculate the relative expression levels of all miRNAs, the $2^{-\Delta \Delta C t}$ cycle threshold method was applied using SNORD44 and hFOB 19.9 or L87/4 for normalization purposes [14].

For validation of selected regulators and targets of the analysed miRNAs, the relative expression levels of TP53 (probe Hs01034249_m1), MYC (probe Hs00153408_m1), E2F1 (probe Hs00153451_m1), E2F2 (probe Hs00231667_m1), E2F3 (probe Hs00605457 $\mathrm{m} 1$ ), FAS (probe Hs00236330_m1), and BIM (probe Hs00708019_s1) were determined by using the TaqMan ${ }^{\circledR}$ Probe-Based Gene Expression Analysis (Applied Biosystems, Life Technologies, Switzerland). The 18S housekeeping probe and hFOB 19.9 or L87/4 cells were used for normalization. All primers were purchased from Applied Biosystems (Life Technologies, Switzerland).

\section{Statistical Analysis}

In order to assess the association between the different relative miRNA expression levels and evaluate their clinical significance we conducted an exploratory statistical analysis. Statistical differences between groups were calculated using a Wilcox-rank sum test and specific cut-offs for grouping the variables into "low" and "high" groups were calculated by maximizing the log rank score in a time dependent survival analysis. The significance level was set to 0.05 . The statistical analysis was done using R Version 3.0.1 [15], including different packages: "MASS" [16]; "coin" [17]; "survival” [18, 19].

\section{Funding}

Daniel Baumhoer and Gernot Jundt were supported by the Forschungsfonds of the University of Basel (DB) and by the Foundation for the Preservation of the Basel Bone Tumor Reference Center (DB and GJ). Jan Smida and Michaela Nathrath belong to the Translational Sarcoma Research Network supported by the BMBF (FKZ01GM0870).

\section{Ethical approval}

The study was approved by the local ethical committee of Basel, Switzerland (EKBB, Reference 274/12).

\section{REFERENCES}

1. Rosenberg AE, Cleton-Jansen A-M, de Pinieux G, Deyrup AT, Hauben E and Squire J. (2013). Conventional osteosarcoma. In: Fletcher CDM, Bridge JA, Hogendoorn PCW and Mertens F, eds. WHO Classification of Tumours of Tumours of Soft Tissue and Bone. (Lyon, France: IARC Press), pp. 282-288.

2. Bielack SS, Kempf-Bielack B, Delling G, Exner GU, Flege S, Helmke K, Kotz R, Salzer-Kuntschik M, Werner M, Winkelmann W, Zoubek A, Jurgens H and Winkler K. Prognostic factors in high-grade osteosarcoma of the extremities or trunk: an analysis of 1,702 patients treated on neoadjuvant cooperative osteosarcoma study group protocols. J Clin Oncol. 2002; 20(3):776-790.

3. Ferrari S, Smeland S, Mercuri M, Bertoni F, Longhi A, Ruggieri P, Alvegard TA, Picci P, Capanna R, Bernini G, Muller C, Tienghi A, Wiebe T, Comandone A, Bohling T, Del Prever AB, et al. Neoadjuvant chemotherapy with high-dose Ifosfamide, high-dose methotrexate, cisplatin, and doxorubicin for patients with localized osteosarcoma of the extremity: a joint study by the Italian and Scandinavian Sarcoma Groups. J Clin Oncol. 2005; 23(34):8845-8852.

4. Kong $\mathrm{C}$ and Hansen MF. Biomarkers in osteosarcoma. 
Expert Opin Med Diagn. 2009; 3(1):13-23.

5. He L, Thomson JM, Hemann MT, Hernando-Monge E, Mu D, Goodson S, Powers S, Cordon-Cardo C, Lowe SW, Hannon GJ and Hammond SM. A microRNA polycistron as a potential human oncogene. Nature. 2005; 435(7043):828833.

6. Xiang J and $\mathrm{Wu}$ J. Feud or Friend? The Role of the miR17-92 Cluster in Tumorigenesis. Current genomics. 2010; 11(2):129-135.

7. Zhou M, Ma J, Chen S, Chen X and Yu X. MicroRNA-17-92 cluster regulates osteoblast proliferation and differentiation. Endocrine. 2013.

8. Li M, Guan X, Sun Y, Shu X and Li C. miR-92a family and their target genes in tumorigenesis and metastasis. Exp Cell Res. 2014.

9. Mogilyansky E and Rigoutsos I. The miR-17/92 cluster: a comprehensive update on its genomics, genetics, functions and increasingly important and numerous roles in health and disease. Cell Death Differ. 2013; 20(12):1603-1614.

10. Baumhoer D, Zillmer S, Unger K, Rosemann M, Atkinson MJ, Irmler M, Beckers J, Siggelkow H, von Luettichau I, Jundt G, Smida J and Nathrath M. MicroRNA profiling with correlation to gene expression revealed the oncogenic miR17-92 cluster to be up-regulated in osteosarcoma. Cancer Genet. 2012; 205(5):212-219.

11. Namløs HM, Meza-Zepeda LA, Baroy T, Ostensen IH, Kresse SH, Kuijjer ML, Serra M, Burger H, Cleton-Jansen $\mathrm{AM}$ and Myklebost O. Modulation of the osteosarcoma expression phenotype by microRNAs. PLoS One. 2012; 7(10):e48086

12. Yang $\mathrm{J}$ and Zhang $\mathrm{W}$. New molecular insights into osteosarcoma targeted therapy. Curr Opin Oncol. 2013; 25(4):398-406.

13. Huang G, Nishimoto K, Zhou Z, Hughes D and Kleinerman ES. miR-20a encoded by the miR-17-92 cluster increases the metastatic potential of osteosarcoma cells by regulating Fas expression. Cancer Res. 2012; 72(4):908-916.

14. Livak KJ and Schmittgen TD. Analysis of relative gene expression data using real-time quantitative PCR and the 2(-Delta Delta C(T)) Method. Methods. 2001; 25(4):402408.

15. R Core Team. (2013). R: A language and environment for statistical computing. R Foundation for Statistical Computing, Vienna, Austria. URL http://www.R-project. org/.

16. Venables WN and Ripley BD. (2002). Modern Applied Statistics with S: Springer, New York.

17. Hothorn T, Hornik K, van de Wiel MA and Zeileis A. A Lego System for Conditional Inference. The American Statistician. 2006; 60(3):257-263.

18. Therneau TM. (2013). A Package for Survival Analysis in S. R package version 2.37-4. URL http://CRAN.R-project. org/package $=$ survival.

19. Therneau TM and Grambsch PM. (2000). Modeling
Survival Data: Extending the Cox Model: Springer, New York.

20. Olive V, Li Q and He L. mir-17-92: a polycistronic oncomir with pleiotropic functions. Immunol Rev. 2013; 253(1):158166.

21. Smida J, Baumhoer D, Rosemann M, Walch A, Bielack S, Poremba C, Remberger K, Korsching E, Scheurlen W, Dierkes C, Burdach S, Jundt G, Atkinson MJ and Nathrath M. Genomic alterations and allelic imbalances are strong prognostic predictors in osteosarcoma. Clin Cancer Res. 2010; 16(16):4256-4267.

22. Wang Z, Liu M, Zhu H, Zhang W, He S, Hu C, Quan L, Bai $\mathrm{J}$ and $\mathrm{Xu} \mathrm{N}$. miR-106a is frequently upregulated in gastric cancer and inhibits the extrinsic apoptotic pathway by targeting FAS. Mol Carcinog. 2013; 52(8):634-646.

23. Guo L, Xu J, Qi J, Zhang L, Wang J, Liang J, Qian N, Zhou H, Wei L and Deng L. MicroRNA-17-92a upregulation by estrogen leads to Bim targeting and inhibition of osteoblast apoptosis. J Cell Sci. 2013; 126(Pt 4):978-988.

24. Meyerson M and Pellman D. Cancer genomes evolve by pulverizing single chromosomes. Cell. 2011; 144(1):9-10.

25. Stephens PJ, Greenman CD, Fu B, Yang F, Bignell GR, Mudie LJ, Pleasance ED, Lau KW, Beare D, Stebbings LA, McLaren S, Lin ML, McBride DJ, Varela I, Nik-Zainal S, Leroy $\mathrm{C}$, et al. Massive genomic rearrangement acquired in a single catastrophic event during cancer development. Cell. 2011; 144(1):27-40.

26. Feugeas O, Guriec N, Babin-Boilletot A, Marcellin L, Simon P, Babin S, Thyss A, Hofman P, Terrier P, Kalifa C, Brunat-Mentigny M, Patricot LM and Oberling F. Loss of heterozygosity of the RB gene is a poor prognostic factor in patients with osteosarcoma. J Clin Oncol. 1996; 14(2):467472.

27. Araki N, Uchida A, Kimura T, Yoshikawa H, Aoki Y, Ueda T, Takai S, Miki T and Ono K. Involvement of the retinoblastoma gene in primary osteosarcomas and other bone and soft-tissue tumors. Clin Orthop Relat Res. 1991; (270):271-277.

28. Jin HY, Lai M and Xiao C. microRNA-17 92 is a powerful cancer driver and a therapeutic target. Cell Cycle. 2014; 13(4). 